\title{
POSITIVE SEMIGROUPS OF OPERATORS ON BANACH SPACES
}

\author{
K. F. NG
}

(Received 21 October 1987)

Communicated by R. O. Vyborny

\begin{abstract}
We prove a version of the Feller-Miyadera-Phillips theorem characterizing the infinitesimal generators of positive $C_{0}$-semigroups on ordered Banach spaces with normal cones. This is done in terms of $N(A)$ as well as the canonical half-norms of Arendt Chernoff and Kato defined by $N(a)=\inf \{\|b\| \mid b \geq a\}$, where $N(A)=\sup \{N(A a) \mid N(a) \leq 1\}$ for operator $A$. A corresponding result on $C_{0}^{*}$-semigroups is also given.

1980 Mathematics subject classification (Amer. Math. Soc.) (1985 Revision): 47 D 05, 47 H 07 , 47 B 44, 46 A 40.

Let $\left(B, B_{+},\|\|\right)$be an ordered Banach space with proper closed convex cone $B_{+}$. The dual $B^{*}$ is ordered by $B_{+}^{*}=\left\{f \in B^{*} \mid f(b) \geq 0\right.$ for all $\left.b \in B_{+}\right\}$. As in [1], [3], [6] and [7], the canonical half-norm $N$ by $N(a)=\inf \{\|b\| \mid a \leq b\}$ for $a \in B$. For a linear operator $A$ from $B$ into itself, we define $N(A)=$ $\sup \{N(A x) \mid N(x) \leq 1\}$. We extend some recent results of Robinson [8], [9] by proving the following analog of the Feller-Miyadera-Phillips theorem (see [2] and [4]).
\end{abstract}

THEOREM 1. Suppose $B_{+}$is normal. Let $H$ be a closed linear operator with domain $D(H)$, a dense subspace of $B$. Then, for constants $M, \omega$, the following statements are equivalent.

(i) $H$ generates a $C_{0}$-semigroup $\left\{S_{t}\right\}$ (so $S_{t}=e^{-t H}$ ) with $S_{t} \geq 0$ (that is $\left.S_{t}\left(B_{+}\right) \subseteq B_{+}\right)$and $N\left(S_{t}\right) \leq M e^{\omega t}, t \geq 0$.

(C) 1989 Australian Mathematical Society $0263-6115 / 89 \$ A 2.00+0.00$ 
(ii) For all small $\alpha>0,(I+\alpha H)^{-1}$ exists and is a positive linear operator on $B$ such that

$$
N\left((I+\alpha H)^{-n} x\right) \leq M(1-\alpha \omega)^{-n} N(x)
$$

for all $x \in B, n \geq 1$.

(iii) The range $R(I+\alpha H)=B$ and

$$
N\left((I+\alpha H)^{n} a\right) \geq(1-\alpha \omega)^{n} N(a) / M
$$

for all $a \in D\left(H^{n}\right), n \geq 1$, and for all small $\alpha>0$.

The equivalence of (ii) and (iii) follows easily from the closed graph theorem and the fact that $N(a)=0$ if and only if $a \leq 0$. For (iii) $\Rightarrow$ (i), we use a suggestion in [1, Remark 4.2]: let $\|a\|_{N}=N(a)+N(-a)$. Then \|\|$_{N}$ is a norm on $B$ equivalent to the given norm \|\| , because $B_{+}$is assumed to be normal. The $N$-dissipative condition in (iii) implies the \|\|$_{N}$-dissipative condition:

$$
\left\|(1+\alpha H)^{n} a\right\|_{N} \geq(1-\alpha \omega)^{n}\|a\|_{N} / M .
$$

By the Feller-Miyadera-Phillips Theorem, $H$ is the infinitesimal generator of a $C_{0}$-semigroup $\left\{S_{t}\right\}$, and $S_{t} x=\lim _{n \rightarrow \infty}(I+(t / n) H)^{-n} x$ for all $x \in B$. Since each $(I+(t / n) H)^{-n} \geq 0$ by (ii), it follows that $S_{t} \geq 0$. Also, by continuity of $N$, it follows from the $N$-dissipativity in (ii) that

$$
N\left(S_{t} x\right) \leq \lim _{n \rightarrow \infty}\left[M\left(1-\frac{t}{n} \omega\right)^{-n} N(x)\right]=M e^{t \omega} N(x)
$$

for all $x \in B$. This shows that $N\left(S_{t}\right) \leq M e^{t \omega}$. Conversely, if (i) holds then, by the standard theory, $(I+\alpha H)^{-1}$ exists and is a continuous linear operator on $B$ such that

$$
(I+\alpha H)^{-n} x=\int_{0}^{\infty}\left(S_{\alpha t} x\right) \frac{t^{n-1}}{(n-1) !} e^{-t} d t .
$$

Since $S_{\alpha t} \geq 0$ it follows that $(1+\alpha H)^{-n} \geq 0$. Also, since $N$ is convex and positively homogeneous, one has, by the following lemma and (i), that

$$
\begin{aligned}
N\left((1+\alpha H)^{-n} x\right) & \leq \int_{0}^{\infty} N\left(S_{\alpha t} x\right) \frac{t^{n-1}}{(n-1) !} e^{-t} d t \\
& \leq \int_{0}^{\infty} N\left(S_{\alpha t}\right) N(x) \frac{t^{n-1}}{(n-1) !} e^{-t} d t \\
& \leq \int_{0}^{\infty} M e^{\alpha \omega t} N(x) \frac{t^{n-1}}{(n-1) !} e^{-t} d t \\
& =M N(x)(1-\alpha \omega)^{-n}
\end{aligned}
$$

proving (i) $\Rightarrow$ (ii) 
LEMMA 1. Let $A$ be a linear operator on $B$ and $\gamma \in \mathbf{R}, \gamma>0$. The following statements are equivalent:

(i) $N(A) \leq \gamma$;

(ii) $N(A x) \leq \gamma N(x)$ for all $x \in B$.

We omit the proof of this easy lemma.

REMARK. If $N(A)<+\infty$ then $A \geq 0$.

Lemma 2. Suppose \|\| is monotone on $B$ and on the dual $B^{*}$, and let $A$ be a positive linear operator on $B$. Then

$$
N(A)=\operatorname{Sup}\{N(A a) \mid a \geq 0, N(a) \leq 1\}=\|A\|_{+}
$$

where $\|A\|_{+}$is the Robinson norm of $A$ and is defined in [9] by

$$
\|A\|_{+}=\sup \{\|A a\| \mid a \geq 0,\|a\| \leq 1\} .
$$

Proof. Since \|\| is monotone on $B, N(a)=\|a\|$ for $a \in B_{+}$. Since \|\| is monotone on $B^{*}, N(a)=\inf \{\|b\| \mid b \geq a, 0\}$ for all $a \in B$ (see [7, Theorem 2.4], and also [5, Proposition 6]). Hence the second equality in (1) is clear. Moreover, for $a \in B$ with $N(a) \leq 1$,

$$
\begin{aligned}
N(A a) & =\inf \{\|c\| \mid c \geq A a, 0\} \\
& \leq \inf \{\|A b\| \mid b \geq a, 0\} \leq \inf \left\{\|A\|_{+}\|b\| \mid b \geq a, 0\right\} \\
& =\|A\|_{+} N(a) \leq\|A\|_{+}
\end{aligned}
$$

which shows that $N(A) \leq\|A\|_{+}$. That $N(A) \geq\|A\|_{+}$holds trivially in view of the second equality in (1). This completes our proof.

Note. In view of this lemma, Theorem 1 , in the special case when \|\| is monotone on $B$ and $B^{*}$, is exactly the same as the theorem of Robinson [9, Theorem 1.1] which in turn generalizes [8, Theorem 3.5], and results in [1], [3] (extensions in line of Theorem 1 were also anticipated in [2, page 264] with less specific bounds). Likewise, our Theorem 2 below was given by Robinson [9], [8] for the special case stated. The following duality result will be important for our discussion of $C_{0}^{*}$-version of Theorem 1 .

LEMMA 3. Suppose $\left(B, B_{+},\|\|\right)$is the dual of an ordered Banach space $\left(B_{*}, B_{*+},\|\|\right)$ with closed convex cone $B_{*+}$. Let $A \in \mathscr{L}(B)$ be the dual of an operator $A_{*} \in \mathscr{L}\left(B_{*}\right)$. Then (i) $A \geq 0$ if and only if $A_{*} \geq 0$,

(ii) $N\left(A_{*}\right)=\|A\|_{+}$, if $A \geq 0$.

Proof. As (i) is well known and easy to verify, we only prove (ii). General elements of $B_{*}$ and $B$ will usually be denoted by $x$ and $f$ respectively. By 
[7, Theorem 2.1],

$$
\begin{aligned}
N\left(A_{*} x\right) & =\sup \left\{f\left(A_{*} x\right) \mid f \geq 0,\|f\| \leq 1\right\} \\
& =\sup \{(A f)(x) \mid f \geq 0,\|f\| \leq 1\} \\
& \leq \sup \left\{g(x) \mid g \in B, g \geq 0,\|g\| \leq\|A\|_{+}\right\} \\
& =\|A\|_{+} N(x),
\end{aligned}
$$

which shows that $N\left(A_{*}\right) \leq\|A\|_{+}$. Here we have used the fact that if $g=A f$ with $f \geq 0$ and $\|f\| \leq 1$ then $g \geq 0$ and $\|g\| \leq\|A\|_{+}\|f\| \leq\|A\|_{+}$.

On the other hand, for $f \geq 0,\|f\| \leq 1$, one has

$$
\begin{aligned}
\|A f\| & =\sup \{(A f)(x) \mid\|x\| \leq 1\} \\
& =\sup \left\{f\left(A_{*} x\right) \mid\|x\| \leq 1\right\} \\
& \leq \sup \left\{N\left(A_{*} x\right) \mid\|x\| \leq 1\right\} \\
& \leq \sup \left\{N\left(A_{*}\right) N(x) \mid\|x\| \leq 1\right\} \\
& \leq N\left(A_{*}\right),
\end{aligned}
$$

which shows that $\|A\|_{+} \leq N\left(A_{*}\right)$. Here [7, Theorem 2.1] has been used again.

TheOREM 2. Let $\left(B, B_{+},\|\|\right)$and $\left(B_{*}, B_{*+},\|\|\right)$ be as in Lemma 3. Suppose $B=B_{+}-B_{+}$. Let $H$ be a $w^{*}$-closed linear operator with domain $D(H) a w^{*}$ dense subspace of $B$. The following conditions are equivalent.

(i) $H$ generates a $C_{0}^{*}$-semigroup $\left\{S_{t}\right\}$ with $S_{t} \geq 0$ and $\left\|S_{t}\right\|_{+} \leq M e^{\omega t}, t \geq 0$.

(ii) For all small $\alpha>0,(I+\alpha H)^{-1}$ exists such that

$$
\left\|(I+\alpha H)^{-n} f\right\| \leq M(1-\alpha \omega)^{-n}\|f\|
$$

for all $f \in B_{+}, n \geq 1$.

Proof. We note first that since $B=B_{+}-B_{+}$, the cone $B_{*+}$ is normal in $B$. Since (2) is equivalent to

$$
\left\|(I+\alpha H)^{-n}\right\|_{+} \leq M(1-\alpha \omega)^{-n}
$$

the proof of (i) $\Rightarrow$ (ii) is the same as that given in [8, Theorem 3.4] and [9, Theorem 1.2]. Conversely, if (ii) holds then, by Lemma $3,(I+\alpha H)_{*}^{-n}=$ $\left(I+\alpha H_{*}\right)^{-n}$ is a positive continuous linear operator on $B_{*}$ such that

$$
N\left(\left(I+\alpha H_{*}\right)^{-n}\right) \leq M(1-\alpha \omega)^{-n}
$$

for all $n$ and all small $\alpha>0$, where $H_{*}$ is norm-densely defined, normedclosed adjoint of $H$ on $B_{*}$. By Theorem 1 applied to $H_{*}$ and $B_{*}$, we conclude that $H_{*}$ generates a $C_{0}$-semigroup $\left\{S_{t}^{*}\right\}$ on $B_{*}$ with $S_{t}^{*} \geq 0$ and $N\left(S_{t}^{*}\right) \leq M e^{\omega t}$, $t \geq 0$. Then $H$ generates the dual semigroup $\left\{S_{t}\right\}$ of $\left\{S_{t}^{*}\right\}$. Furthermore, by Lemma $3, S_{t} \geq 0$ and $\left\|S_{t}\right\|_{+}=N\left(S_{t}^{*}\right) \leq M e^{\omega t}$ for all $t \geq 0$. 
REMARK. In the special case $M=1$ and $\omega=0$, Theorem 1 corresponds to the Hille-Yosida theorem, that is, $S$ is $N$-contractive (in the sense that $N\left(S_{t}\right) \leq 1$ for all $\left.t\right)$. The dissipative condition in (iii) then reduces to the single condition $N((I+\alpha H) a) \geq N(a)$ because the higher order conditions follow by iteration. Similarly, for $M=1$ and $\omega=0$, Theorem 2 simply states that $H$ generates a $C_{0}^{*}$-semigroup of positive \|\|$_{+}$-contractions if and only if $(I+\alpha H)^{-1}$ is a positive $w^{*}$-continuous \|\|$_{+}$-contraction for all small $\alpha>0$.

\section{References}

[1] W. Arendt, P. R. Chernoff, and T. Kato, 'A generalization of dissipativity and positive semigroups', J. Operator Theory 8 (1982), 167-180.

[2] C. J. K. Batty and D. W. Robinson, 'Positive one-parameter semigroups on ordered Banach spaces', Acta Appl. Math. 1 (1984), 221-296.

[3] O. Bratteli, T. Digernes and D. W. Robinson, 'Positive semigroups on ordered Banach spaces', J. Operator Theory 9 (1983), 371-400.

[4] E. B. Davies, One-parameter semigroups, (Academic Press, London, 1980).

[5] K. F. Ng, 'The duality of partially ordered Banach spaces', Proc. London Math. Soc. 19 (1969), 269-288.

[6] D. W. Robinson and S. Yamamuro, 'The Jordan decomposition and half-norms', Pacific J. Math. 110 (1984), 345-353.

[7] D. W. Robinson and S. Yamamuro, 'The canonical half-norm, dual half-norms and monotonic norms', Tôhoku Math. J. 35 (1983), 375-386.

[8] D. W. Robinson, 'Continuous semigroups on ordered Banach Spaces', J. Funct. Anal. 51 (1983), 268-284.

[9] D. W. Robinson, 'On positive semigroups', Publ. RIMS Kyoto University 20 (1984), 213224.

\section{Department of Mathematics \\ Chinese University of Hong Kong \\ Hong Kong}

